\title{
Osteogenic Differentiation of Pre-Conditioned Bone Marrow Mesenchymal Stem Cells with Nisin on the Modified Poly-L-Lactic-Acid Nanofibers
}

\section{Fariba Sadraei}

Islamic Azad University Tehran Medical Sciences

Marzieh Ghollasi

Kharazmi University - Karaj

Fatemeh Khakpai

Islamic Azad University Tehran Medical Sciences

Raheleh Halabian ( $\nabla$ r.halabian@yahoo.com)

Baqiyatallah University of Medical Sciences https://orcid.org/0000-0002-3363-8276

\section{Nano Express}

Keywords: Differentiation, Osteogenesis, Mesenchymal Stem Cell, PLLA scaffold, Nisin, Preconditioning

Posted Date: June 7th, 2021

DOI: https://doi.org/10.21203/rs.3.rs-553590/v1

License: (c) This work is licensed under a Creative Commons Attribution 4.0 International License. Read Full License 


\section{Abstract}

Background: Human bone marrow-derived mesenchymal stem (MSCs) cells are undifferentiated cells with the self-renewing ability and multi-lineage differentiation beneficial for regenerative medicine. Nano scaffolds are novel materials employed in bone repair and regeneration. Nisin is a prebiotic that can increase stem cells' life span and proliferation. This study attempted to provide a proper strategy for bone marrow mesenchymal stem cells differentiation into the Osteocytes on a Poly-L-lactic-acid scaffold (PLLA) after pretreating with probiotic Nisin.

Methods: MSC osteogenic differentiation was evaluated by measuring Calcium, Alkaline phosphatase, and quantitative tests such as Real-Time PCR, Acridine Orange, Alizarin Red, Von Kossa, and others.

Results: The result of the MTT test showed that the optimal dose of Nisin probiotic for the MSCs' preconditioning was $200 \mathrm{lU} / \mathrm{mL}$ on the 1st, 3rd, and 5th days of culture. Real-time PCR data indicated that the expression rate of ALP, Osteonectin, Osteocalcin, and Collagen I have increased in the presence of Nisin, while the RUNX-2 gene expression has decreased. Furthermore, the results of Alizarin Red and Von Kossa tests, as well as Scanning electron microscopy (SEM), revealed that the cell proliferation in the preconditioned samples with Nisin increased significantly.

Conclusions: The study concluded that the cell proliferation and differentiation increased in samples pretreated with Nisin on the PLLA Nano scaffolds.

\section{Introduction}

Nano scaffolds are novel materials take in repairing and bone regeneration. Poly-L-lactic-acid (PLLA) is a synthetic nanofiber polymer with biodegradable, biocompatible, and permeability (porosity) properties (1, 2). PLLA scaffold is a component of Poly-a-hydroxy esters with hydrophilic, cell adhesion, and cell deformation properties $(3,4)$, with an intrinsic viscosity of $1.6 \mathrm{dl} / \mathrm{g}(5)$. PLLA carries proliferated and differentiated cells as a biocompatible tissue supporting matrix that gradually biodegraded after a specified time. This scaffold possesses high mechanical strength, so it is suitable for employing bone tissue repair $(1,4)$.

Human bone marrow-derived mesenchymal stem cells (hMSCs) are capable of self-renewing and differentiating into three cell lines (Osteocytes, Chondrocytes, and Adipocytes) with proper in vitro induction $(3,6,7)$. MSCs are safe for allogeneic transplantation due to lack of MHCII expression on the surface and consequent lack of intense immune responses. MSCs' unique characteristics make them favorable candidates for tissue engineering, while their short life span in harsh conditions is considered a limitation. Recently, to overcome this problem, preconditioning strategies in stem cell therapy have been suggested. For this issue, MSCs are pretreated with some compounds or conditions which not only increase their life span but they also occasionally improve their proliferation and differentiation. The compounds used for preconditioning including some hazardous materials such as toxins, $\mathrm{H}_{2} \mathrm{O}_{2}$, hypoxic 
conditions, etc., or many beneficial compounds such as growth factors, cytokines, pre/probiotics, etc. (5, 8-10).

Nisin is a small antimicrobial peptide with 34 amino acids belonging to the Lantibiotic group. The presence of dehydrated residues and Lanthionine rings tightens Nisin's peptide structure, which makes it more attractive. There are various types of Nisin, as Nisin A, Z, F, Q, H, and others (11).

Based on the mentioned evidence, the present study aimed to see whether a short time preconditioning of MSCs with a low concentration of Nisin as a probiotic and culturing on the PLLA Nano scaffold would stimulate the proliferation and osteogenic differentiation.

\section{Results}

\subsection{Results of MTT Assay}

An optimal and non-lethal optimum for the MTT test was performed to determine the optimum dosage of Nisin for MSC pre-conditioning on days 1, 3, and 5. The optimum dosage of Nisin, that survived more than $80 \%$ of cells was $200 \mathrm{IU} / \mathrm{mL}$. It was observed that this dose was appropriate for cell viability and there was no significant difference between groups (Fig. 1).

\subsection{Results of Acridine Orange Staining}

The cell apoptosis and growth and proliferation of MSCs were performed qualitatively with ocher Acridine staining on days 1, 3, and 5. It was observed that the MSCs on the PLLA scaffold and the Nisin preconditioning MSCs had almost normal growth and proliferation comparing to the control group, and none of them increased the apoptosis rate in the MSCs (Fig. 2).

\subsection{Results of Alkaline Phosphate Enzyme activity}

Determination of alkaline phosphatase activity on the days 7th and 14th of osteogenic differentiation of MSCs was performed. The level of alkaline phosphatase enzyme activity did not differ significantly between the scaffold and Nisin groups, and control on day 7th. But on day 14th, the differentiation in the scaffold and Nisin groups showed a significant difference in comparison to the control group $(P \leq 0.01)$ (Fig. 3).

\subsection{Results of Calcium Content}

Calcium sedimentation in differentiated bone marrow cells was distinguished on the days 7 th and 14 th. On day 7th, differentiation in the scaffold and Nisin groups was significantly increased when compared with the control group $(P \leq 0.05)$, showing calcium sedimentation. On day 14 th, differentiation of calcium sedimentation rate in scaffolds and Nisin had a significant difference with the control group $(P \leq 0.01)$. Usually, a conclusion is weighted in the discussion section (Fig. 4).

\subsection{Alizarin Red and Von Kossa Staining}


Calcium and mineral sediments were evaluated using Von Kossa coloring on the 14th day of differentiation. It was observed that in the scaffold group and Nisin group, the calcium sedimentation rate increased compared with the control group (Fig. 5). In the calcium sedimentation study, using Alizarin Red staining, the amount of minerals in the scaffold group and Nisin group is more than the control group (Fig. 5). Von Kossa and Alizarin Red staining confirmed the osteogenic differentiation in BM-MSCs in the PLLA + NI sample.

\subsection{Morphological study of the differentiated cells}

The morphology, adhesion, and biocompatibility of BM-MSCs on the PLLA scaffold were investigated on the day 14th of differentiation. The amount of adhesion of the PLLA scaffold was confirmed (Fig. 6C), as well as the number of differentiated cells on the scaffold and Nisin samples were compared to the scaffold group (Fig. 6D).

\subsection{Immunocytochemical staining of differentiated BM- MSCs}

A qualitative study of Osteocalcin and Osteopontin proteins was investigated on the day 14th of differentiation. To confirm the differentiation of BM-MSCs, immunofluorescence staining was used. The levels of Osteocalcin and Osteopontin proteins in differentiated cells in the scaffold and Nisin groups increased compared to the control group (Fig. 7A, B).

\subsection{Real-time PCR analysis}

To investigate the expression of alkaline phosphatase, Osteocalcin, Osteonectin, collagen type 1, and RUNX-2, on days 7 and 14, the differentiation of BM-MSCs was done using Real-Time PCR. On day 7th (Fig. 8A), the gene expression of alkaline phosphatase as a marker of differentiation increased in the MSN-P-N and MSC-N group compared to the MSCs (control) group $(P \leq 0.01)$. Moreover, the Osteonectin gene expression was significantly increased in the MSC-P-N group when compared to the control group $(P \leq 0.01)$. Interestingly, the gene expression of Osteocalcin was elevated 5-times in the MSC-P-N group than that of the control group. The expression of the collagen type 1 gene was significantly higher in MSC-P-N and MSC-N groups than in the control group ( $P \leq 0.01)$. On day 14th, the expression of the RUNX-2 gene, as the primary genes in the differentiation, was significantly increased in the MSC-P-N group in comparison with the control group ( $\mathrm{P} \leq 0.01$ ) (Fig. 8B). The distinction between alkaline phosphatase, Osteonectin, Osteocalcin, and type 1 collagen in the MSC-PN group was significantly higher than the control group $(P \leq 0.01)$. On the other hand, the distinct expression of the RUNX-2 gene in all groups decreased on day 7 th .

\section{Discussion}

This study has referred to the effect of Polycarboxylic acid and probiotic Nisin scaffold on the differentiation of human bone marrow mesenchymal stem cells. The concentrations of Nisin examined in this study were 100-200-300 IU/mL. Regarding the MTT test used to test the scaffold toxicity, we find the 
appropriate dosage for preserving mesenchymal stem cells. We observed that the scaffold did not produce any toxicity on mesenchymal stem cells at $200 \mathrm{IU} / \mathrm{mL}$ dosage. Thus, this does select as the appropriate dose of Nisin.

Besides, inspecting the chitosan scaffold by the MTT-based assay revealed that it did not effectively induce the adipocyte MSCs differentiation (2). Checking also out by the MTT test showed non-toxic effects of Polycaprolactone scaffold on hMSCs (8). Moreover, the MTT test showed no toxicity Polycarboxylic acid scaffold for the MSCs differentiation (4). In this study using Alizarin Red staining, it determined that MSCs can produce calcium deposits during differentiation. The results illustrated the process of differentiation by showing calcium deposits secreted from differentiated bone cells. Von Kossa's staining of calcium deposits in the extracellular matrix also confirms that calcium sediments are completely visible after 14 days of differentiation of bone marrow MSCs in osteoblasts (12). In 2016, Paulson and his colleagues reported that calcium deposition in stem cells provides a suitable basis for differentiating MSCs (13). Alkaline phosphatase, which secretes osteoblasts, can contribute to the degradation of mineral pyrophosphate for the organization (1). Our study evaluated the magnitude of alkaline phosphatase on the days 7 th and 14 th. The results showed that alkaline phosphatase activity is the early indicator of osteoporosis. So, utilizing a lactic acid poly-scaffold does not inhibit the secretion of alkaline phosphatase and cell mineralization. Nisin probiotics also have a booster effect on cell differentiation. It increased alkaline phosphatase levels in differentiated cells. On the 14th day of differentiation, this effect was quite distinctive. The next section of the present study investigated the alkaline phosphatase activity in the bone marrow MSCs differentiation on Nano-hydroxyapatite/ collagen and poly-lactic-co-glycolic acid substrate. The data showed that alkaline phosphatase activity reached its maximum in these cases. In addition to markers in bone marrow cells, osteonectin was examined in this study $(14,15)$. In our study, the expression of Osteocalcin, Osteonectin, and collagen type 1 differed significantly on days 7th and 14th. Accordingly, on day 14th, the differentiation of these markers showed a significant increase. RUNX-2 plays an essential role in osteoblasts' development and chondrocyte's maturation. RUNX-2 also increases the binding affinity to DNA and the stability of proteins. Its level remains at the highest level in non-adult osteoblasts, and RUNX-2 decreases when MSCs differentiate into osteoblasts (9).

In another study, the differentiation process was investigated in three groups of bone marrow, fat, and somatic bone marrow MSCs on biochemical nanocomposite scaffolds and Polycaprolactone (6). The obtained results indicated that the amount of calcium accumulation in differentiated bone marrow MSCs was more than that of the two others. Our results also revealed that the calcium accumulation in samples isolated on nanocomposite scaffolds showed the highest than that of control samples. We employed preconditioned bone marrow MSCs with the prebiotic Nisin and PLLA scaffold together. We also examined the mRNA expression of RUNX-2, Osteonectin, Osteocalcin, collagen, and alkaline phosphatase genes. The results presented more gene expression of the RUNX-2 on day 14th rather than the day 7th. Besides, the amount of Osteocalcin expression in this study decreased at the same time (6). Furthermore, the Osteocalcin gene expression in differentiated embryonic MSCs of osteoblast was investigated on 
Nano-hydroxyapatite/ collagen/ collagen/ polylactic scaffolds (14-16). It determined that the gene expression of this was the highest in the scaffold group than that of other groups.

\section{Conclusion}

The present study concludes that due to the human need several days to repair bone damage, the development of effective scaffolds that can be harmless to the body is necessary. In the present study, the structure of Polycarboxylic acid scaffolds exhibited the potential to support the growth, proliferation, and differentiation of human bone marrow MSCs. Also, it has biodegradability and compatibility with mesenchymal living cells. Besides, bone indices such as alkaline phosphatase activity, calcium sedimentation, and bone marrow gene expression confirmed this evidence. Nisin prebiotic conferred explicit and adequate confirmation, which has positive effects on the differentiation process of bone marrow MSCs.

\section{Material And Methods}

\subsection{Human bone marrow mesenchymal stem cell culture}

The hMSCs used for the experiments were kindly provided by the Stem Cell Technology Research Center (Iran) with the ethical approval certificate. The hMSCs were cultured at a density of about $5 \times 10^{3}$ cells $/ \mathrm{cm}^{2}$ in DMEM high glucose medium, $10 \% \mathrm{FBS}$, and penicillin/streptomycin in an incubator at $37^{\circ} \mathrm{C}$ and $5 \% \mathrm{CO}_{2}$. The cell culture medium was replaced every 48 hours. The hMSCs harvested at almost $80 \%$ confluence of about $2 \times 10^{4}$ cells $/ \mathrm{cm}^{2}$ will result in hMSCs doubling per passage. Following passage 3 , they were characterized to be MSCs by observing spindle-shaped cells, plastic adherence feature, and owning the capability of differentiation to osteogenic cells (the data not shown).

\subsection{Osteogenic differentiation of hMSCs}

For differentiation of MSCs, a differentiation medium has $\beta$-glycerol phosphate $(1.8 \mathrm{~g}$ per $5 \mathrm{ml}$ of deionized water), dexamethasone ( $0.0017 \mathrm{~g}$ per $50 \mathrm{ml}$ culture medium), and ascorbic acid ( $0.19 \mathrm{~g}$ per 20 $\mathrm{ml}$ of culture medium) prepared. Following treatment with differentiation medium, cells maintained at $37^{\circ} \mathrm{C}$ and $5 \% \mathrm{CO}_{2}$ for two weeks in an incubator, and every 48 hours medium was changed.

\subsection{MTT assay}

Three concentrations (100-200-300 IU/mL) of Nisin were evaluated for the optimal Nisin dosage used for the preconditioning of MSCs on the $1 \mathrm{st}, 3 \mathrm{rd}$, and 5th days of culture. In each well of a 48-well plate $1 \times 10^{4}$, MSCs were cultured and incubated in $37^{\circ} \mathrm{C}$ and $5 \% \mathrm{CO}_{2}$. After the $1 \mathrm{st}, 3 \mathrm{rd}$, and 5 th days of culture, $200 \mu \mathrm{l}$ of MTT (3-(4,5-dimethylthiazol-2-yl)-2,5-diphenyltetrazolium bromide) solution was added to each well. Then the plate was incubated for 3 hours. In the next step, $100 \mu \mathrm{l}$ of Dimethyl sulfoxide (DMSO) solution was added and the solution of each well was transferred to a 96-well plate. Then the readings were performed at $540-630 \mathrm{~nm}$ by an ELISA reader. 


\subsection{Acridine Orange Staining}

Acridine Orange staining was performed to investigate the growth and proliferation of MSCs on the 1st, 3rd, and 5th days of culture. 200-300 $\mu$ l of Acridine orange solution was added to each well and incubated for 5 minutes at $37^{\circ} \mathrm{C}$ with $10 \% \mathrm{CO}_{2}$. In the next step, the cells were washed twice with PBS and photographed by the fluorescence microscope.

\subsection{Alkaline Phosphatase Activity}

The activity of alkaline phosphatase as a marker in differentiated bone marrow cells was investigated on the 7th and 14th days. At first, $200 \mu \mathrm{l}$ of Ripa solution was added to each well and the pipette was completely taken and the solution of each well with scaffolds was transferred to $1.5 \mathrm{ml}$ vials and vortexed. The vials were centrifuged $\left(4^{\circ} \mathrm{C}, 15,000 \mathrm{rpm}, 15 \mathrm{~min}\right)$. Using an alkaline phosphatase kit (Pars Azmoon, Tehran, Iran) and adding $150 \mu \mathrm{l}$ of R1 and R2 solutions, plus $50 \mu \mathrm{l}$ of samples, which transferred to a 96-well plate, readings at $450 \mathrm{~nm}$ were carried out by the ELISA reader.

\subsection{Calcium Content Assay}

The amount of calcium sediment accumulation in differentiated MSCs to osteocytes was evaluated on days 7th and 14th of the differentiation. $200 \mu \mathrm{l}$ of the hydrochloric acid solution was added to each well of a 6-well plate. After pipetting, the contents of each well were transferred to $1.5 \mathrm{ml}$ vials and shaken for 40 minutes. Using a Calcium Detection Kit (Pars Azmoon, Tehran, Iran) and adding $150 \mu \mathrm{l}$ of R1 and R2 solutions plus $50 \mu$ l of samples, they were transferred to a 96 -well plate and read out at $570 \mathrm{~nm}$ by the ELISA reader.

\subsection{Alizarin Red Staining}

To assess the quality of calcium deposition, on the day 14th of differentiation was performed. The wells were removed and washed once with PBS and twice with deionized water. 4\% Paraformaldehyde was added to each well and placed at $4^{\circ} \mathrm{C}$ for 20 minutes. In the next step, and Alizarin solution was poured onto cells and scaffolds. Then it was washed with PBS for 5 minutes and photographed under a reverse microscope.

\subsection{Imaging by scanning electron microscopy}

To investigate the morphology, and adhesion as well as biocompatibility of MSCs with PLLA scaffolds, we used scanning electron microscopy.

After 14 days, MSCs were differentiated to the osteocytes all of the wells were washed with PBS. $2.5 \%$ Glutaraldehyde was added to each well and washed twice with PBS for 30 minutes. Then, alcohol dehydration was carried out (60-100-100\%) each well for 10 minutes. The fibers were coated with gold when they dried. Then they were scanned by a scanning electron microscope.

\subsection{Synthesis of Poly-L-lactic acid (PLLA) nanofibers}


Nanoparticles of poly-lactic acid were made using the electrospinning method. 0.43 gr of polymeric polylactic acid was dissolved in $5 \mathrm{~mL}$ of chloroform solution. Then the solution was transferred to the syringe of the device. It was subjected to a voltage of $15 \mathrm{kV}$ with a speed of $0.4 \mathrm{~mL} / \mathrm{h}$. Then, the fibers are made on the aluminum plate.

\subsection{Immunocytochemistry}

To evaluate the Osteocalcin and Osteopontin proteins, on the 14th day of differentiation, the supernatant was removed and cells were washed with PBS. To fix the samples were added, $4 \%$ paraformaldehyde, 20 minutes, $0.4 \%$ Triton X100, 10 minutes, respectively. Then, the goat's serum of $5 \%$, the primary antibodies of Osteocalcin (1:100), and Osteopontin (1:200) were added. After 30 minutes' incubation $\left(37^{\circ} \mathrm{C}, 5 \% \mathrm{CO}\right)$ were washed three times with Phosphate Buffered Saline (PBS). In the next step, the secondary antibody, Fluorescein isothiocyanate (FITC) (1:100), was added and incubated at room temperature for 1 hour. Then, it washed again with PBS. Cell cores were stained with a DAPI (4',6-diamidino-2-phenylindole) color of $0.1 \mu \mathrm{g} / \mathrm{mL}$ and photographed with a fluorescence microscope.

\subsection{Analysis of metastasis genes expression by quantitative RT-PCR analysis}

\subsubsection{RNA extraction}

To determine the expression of alkaline phosphatase, Osteocalcin, Osteonectin, collagen type 1, and RUNX-2 on days 7th and 14th, all tested specimens were firstly subjected to RNA isolation. The supernatant of the wells was discarded and washed with PBS. $700 \mu \mathrm{l}$ of RNA X plus solution was added to each well and placed in an incubator for 10 minutes. The contents of the wells were transferred to a microwave oven, and $200 \mu \mathrm{l}$ of cold chloroform was added to each vial, and centrifugation (12000 rpm, $15 \mathrm{~min}$, at $7^{\circ} \mathrm{C}$ ) was performed. Then $100 \mu \mathrm{l}$ of supernatant was transferred to a new vial and then added $400 \mu \mathrm{l}$ of cold isopropanol. The supernatant was discarded Again, and $1 \mathrm{ml}$ of $75 \%$ alcohol was added to each vial. Then centrifugation (7800 rounds, 10 minutes, low temperature) was performed. After drying completely, $25 \mu$ l of diethyl pyrocarbonate (DEPC) Water was added to each vial and Vortexed. The quality of RNAs was measured by spectrophotometry (NanoDrop, Wilmington, DE). The concentration of RNA was read at $280 / 260 \mathrm{~nm}$ of optical absorption. The proper absorption is between 1.8-2.0, indicating the proper concentration of RNA in the specimens.

\subsection{2. cDNA synthesis}

Appropriate concentrations of RNA were picked up, with about $2 \mu$ l of each primer, $1 \mu$ l oligo-dT, and $1 \mu \mathrm{l}$ random hexamer primer. The obtained solutions were placed in the PCR apparatus at $65^{\circ} \mathrm{C}$ for 5 minutes. Next, $2 \mu$ including RTase Buffer 10X and 0-1MDTT, $1 \mu$ l Reverse Transcriptase, and RNase Inhibitor were 
added, so the final volume reached $20 \mu \mathrm{l}$. The new times and temperatures were set for 5 minutes at $25^{\circ} \mathrm{C}$, 60 minutes at $55^{\circ} \mathrm{C}$, and 4 minutes at $80^{\circ} \mathrm{C}$, for 35 cycles.

\subsubsection{Real-time PCR}

After CDNA synthesis, they were subjected to real-time PCR protocol according to the kit manufacturer's instructions. It is performed using DNA primers and adding SYBER-green qPCR Master Mix. Then the vials are put in a Rotor-gene (Corbett) real-time analyzer. The times and temperature settings included 20 seconds at $95^{\circ} \mathrm{C}, 30$ seconds at $60^{\circ} \mathrm{C}$, and 30 seconds at $72^{\circ} \mathrm{C}$, for 38 cycles.

\subsection{Statistical analysis}

All experiments were repeated three times. The results were reported as mean \pm standard deviation.

Statistical analysis was performed using SPSS software by t-test. P $\leq 0.05$ was considered for significant changes. Real-time PCR data were analyzed using Rotor-Gene Q and REST software.

The data that support the findings of this study are available on request from the corresponding author.

\section{Abbreviations}

MSCs; mesenchymal stem cells

hBM-MSCs; Human bone marrow-derived mesenchymal stem cells

PLLA scaffold; Poly-L-lactic-acid scaffold

SEM; Scanning electron microscopy

MTT; 3-(4,5-dimethylthiazol-2-yl)-2,5-diphenyltetrazolium bromide

DMSO; Dimethyl sulfoxide

PBS; Phosphate Buffered Saline

FITC; Fluorescein isothiocyanate

DAPI; 4',6-diamidino-2-phenylindole

DEPC; diethyl pyrocarbonate

\section{Declarations}

\section{Availability of data and materials}


The datasets used and/or analysed during the current study are available from the corresponding author on reasonable request.

\section{Competing Interest}

All authors declare that they have no conflict of interest.

\section{Funding}

This research did not receive any specific grant from funding agencies in the public, commercial, or notfor-profit sectors.

\section{Authors' Contributions}

Sadraei, Halabian and Khakpai reviewed literature, outlined, wrote the manuscript. Ghollasi and Halabian edited the manuscript and prepared figures. All authors read and approved the final manuscript.

\section{Acknowledgment}

The cells used for experiments were kindly provided by the Stem Cell Technology Research Center (Iran).

\section{References}

1. Vinatier C, Guicheux J. Cartilage tissue engineering: From biomaterials and stem cells to osteoarthritis treatments. Annals of physical and rehabilitation medicine. 2016;59(3):139-44.

2. Ghanbari E, Naseri K, Khazaei M, Akbari M, Elahian F, Mirzaei S, et al. An introduction to tissue engineering and the most widely used scaffolds. Journal of Isfahan Medical School. 2018;36(488).

3. Augello A, De Bari C. The regulation of differentiation in mesenchymal stem cells. Human gene therapy. 2010;21(10):1226-38.

4. Tavangar B, Arasteh S, Edalatkhah H, Salimi A, Doostmohammadi A, Seyedjafari E. Hardystonitecoated poly (I-lactide) nanofibrous scaffold and efficient osteogenic differentiation of adiposederived mesenchymal stem cells. Artificial organs. 2018;42(11):E335-E48.

5. Komori T. Regulation of osteoblast differentiation by transcription factors. Journal of cellular biochemistry. 2006;99(5):1233-9.

6. Hosseini FS, Soleimanifar F, Ardeshirylajimi A, Vakilian S, Mossahebi-Mohammadi M, Enderami SE, et al. In vitro osteogenic differentiation of stem cells with different sources on composite scaffold containing natural bioceramic and polycaprolactone. Artificial cells, nanomedicine, and biotechnology. 2019;47(1):300-7. 
7. Walmsley GG, Ransom RC, Zielins ER, Leavitt T, Flacco JS, Hu MS, et al. Stem cells in bone regeneration. Stem Cell Reviews and Reports. 2016;12(5):524-9.

8. Hoveizi E, Nabiuni M, Parivar K, Massumi M, Ai J. Inductive effect of IDE1 on differentiation of human induced pluripotent stem cells into definitive endoderm cells by using PCL nanofibrous scaffold. Nova Biologica Reperta. 2014;1(1):8-22.

9. Komori T. Regulation of proliferation, differentiation and functions of osteoblasts by Runx2. International journal of molecular sciences. 2019;20(7):1694.

10. Seifert A, Werheid DF, Knapp SM, Tobiasch E. Role of Hox genes in stem cell differentiation. World journal of stem cells. 2015;7(3):583.

11. Field D, Begley M, O'Connor PM, Daly KM, Hugenholtz F, Cotter PD, et al. Bioengineered nisin A derivatives with enhanced activity against both Gram positive and Gram negative pathogens. PloS one. 2012;7(10).

12. Shahrousvand M, Sadeghi GMM, Shahrousvand E, Ghollasi M, Salimi A. Superficial physicochemical properties of polyurethane biomaterials as osteogenic regulators in human mesenchymal stem cells fates. Colloids and Surfaces B: Biointerfaces. 2017;156:292-304.

13. Ding X, Yang G, Zhang W, Li G, Lin S, Kaplan DL, et al. Increased stem cells delivered using a silk $\mathrm{gel} / \mathrm{scaffold}$ complex for enhanced bone regeneration. Scientific reports. 2017;7(1):1-10.

14. Wang X, Zhang G, Qi F, Cheng Y, Lu X, Wang L, et al. Enhanced bone regeneration using an insulinloaded nano-hydroxyapatite/collagen/PLGA composite scaffold. International journal of nanomedicine. 2018;13:117.

15. Chesnutt BM, Yuan Y, Buddington K, Haggard WO, Bumgardner JD. Composite chitosan/nanohydroxyapatite scaffolds induce osteocalcin production by osteoblasts in vitro and support bone formation in vivo. Tissue Engineering Part A. 2009;15(9):2571-9.

16. Wu S, Xiao Z, Song J, Li M, Li W. Evaluation of BMP-2 enhances the osteoblast differentiation of human amnion mesenchymal stem cells seeded on nano-hydroxyapatite/collagen/poly (I-lactide). International journal of molecular sciences. 2018;19(8):2171.

\section{Figures}




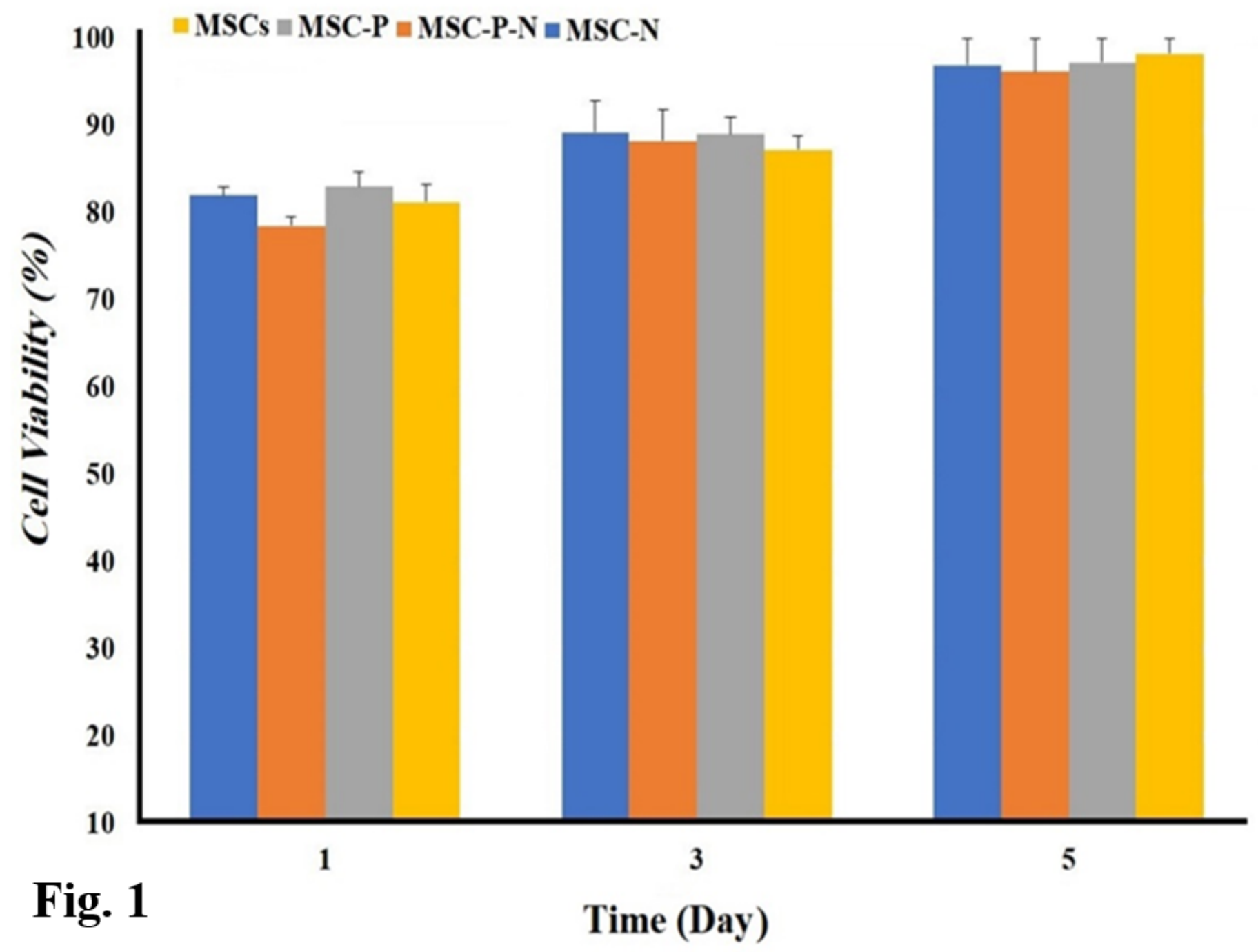

Figure 1

Proliferation of BM-MSCs on scaffold and Nisin and TCPs during 1, 3, and 5 days' culture period 


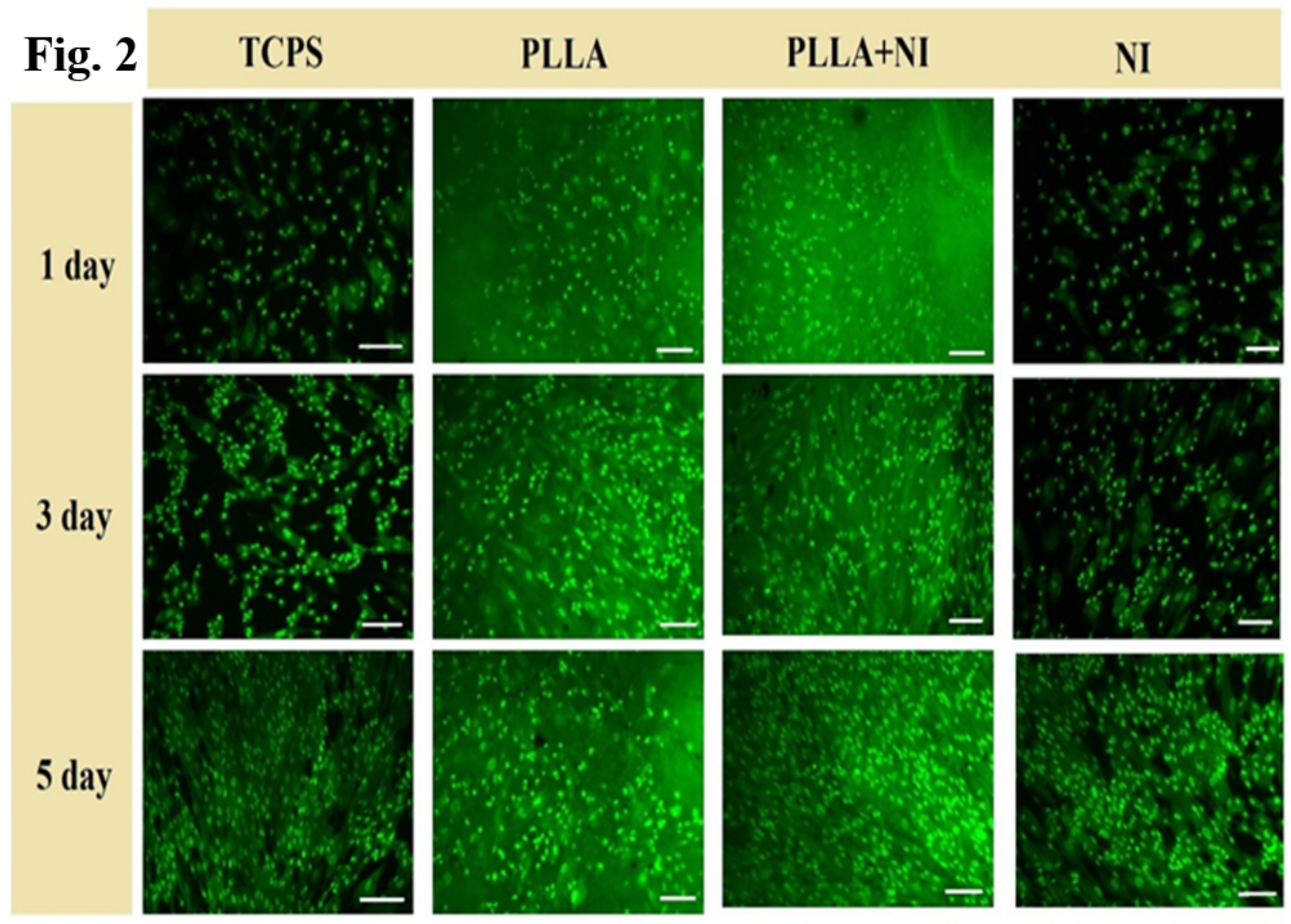

Figure 2

Representative images of cells grown in various wells and stained by Acridine Orange. TCPS (control sample), PLLA nanofibers, PLLA+NI scaffolds, and NI (Nisin). The nucleic and mitochondria appear green, while if cells are damaged or suffering cells should appear red-orange fluorescence colored (Scale bar is $100 \mu \mathrm{m}$ ) 


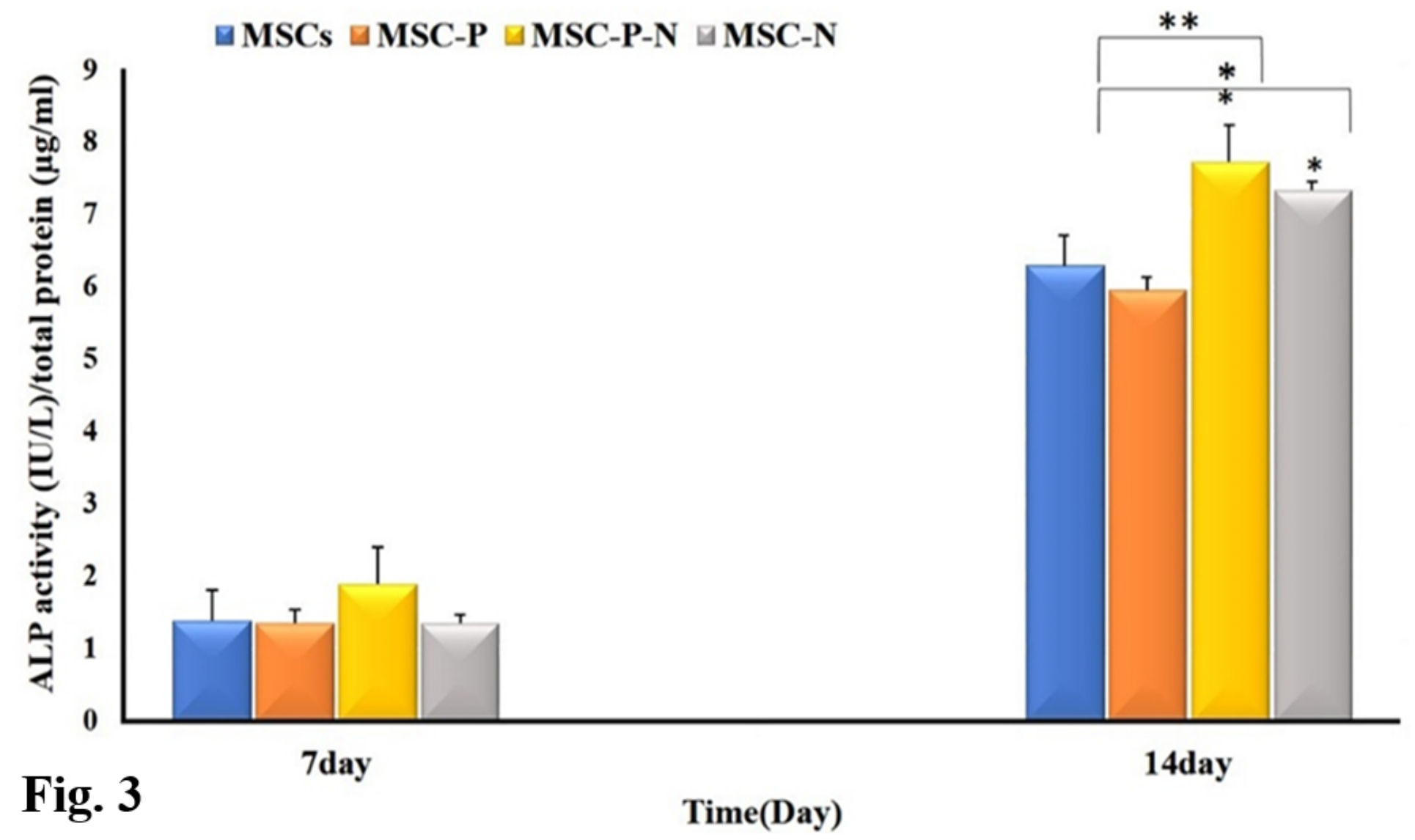

Figure 3

ALP activity of stem cells on PLLA nanofibers scaffold and Nisin probiotic and TCPS during osteogenic differentiation in 7 and 14 days. (Mean $\pm S D ; * P \leq 0.05$ and $* * P \leq 0.01$ ) 


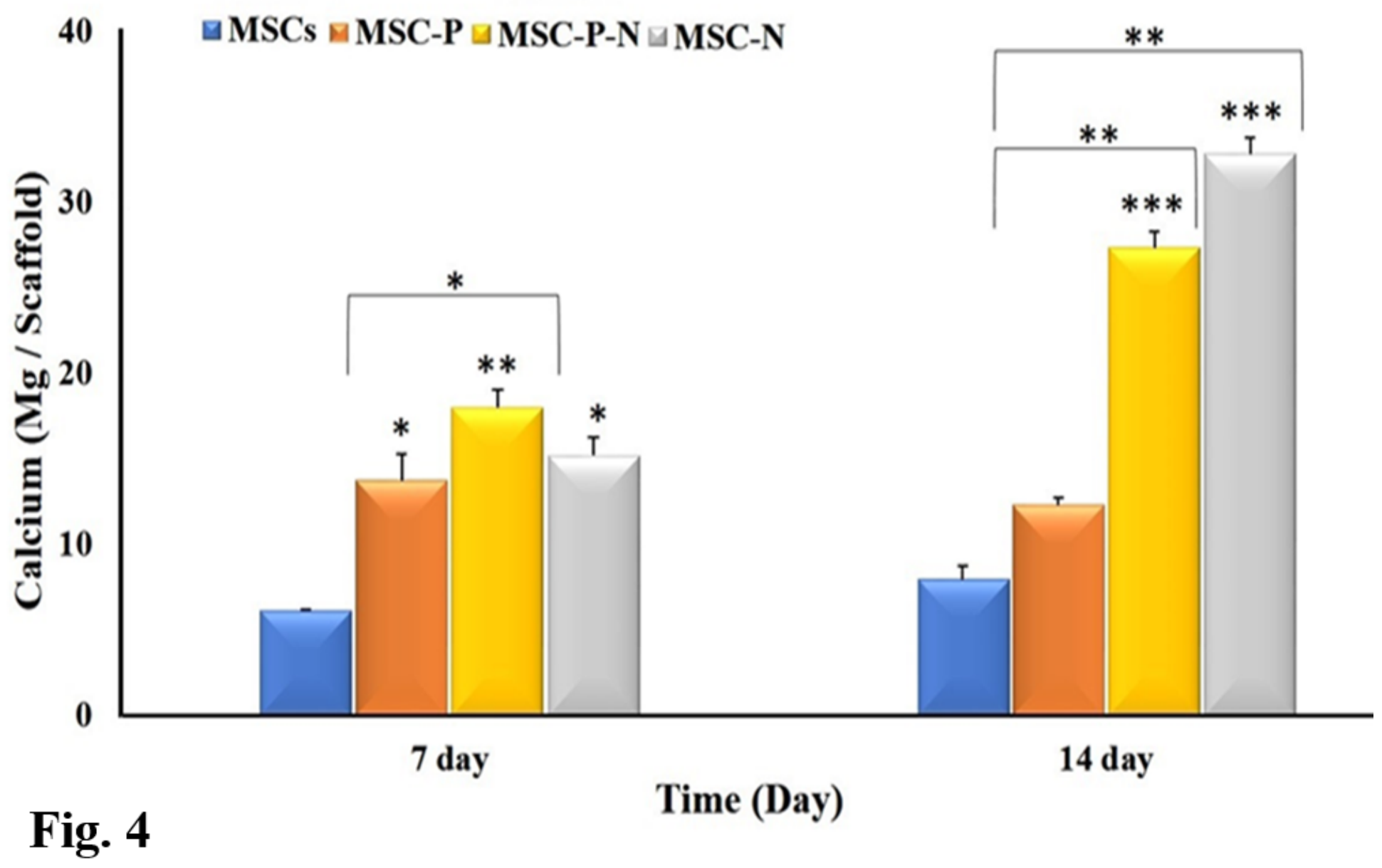

Figure 4

Deposition of Calcium on PLLA nanofibers scaffold and Nisin probiotic and TCPS during osteogenic differentiation in 7 and 14 days. (Mean $\pm S D ; * P \leq 0.05$ and $* * P \leq 0.01$ and $* \star * P \leq 0.001$ ) 


\section{Fig. 5 TCPS PLLA PLLA+NI NI}

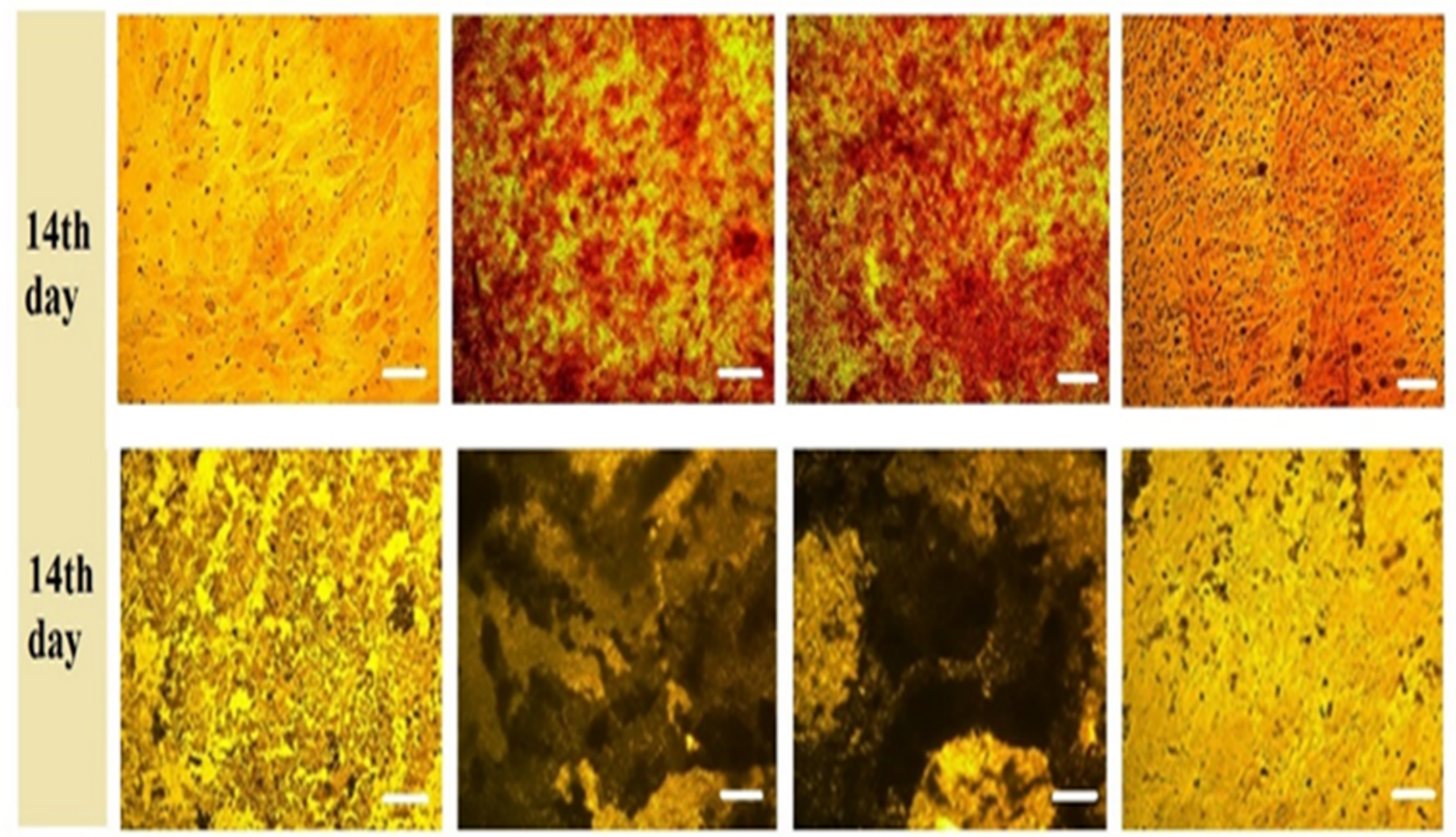

Figure 5

Alizarin Red and Von Kossa Staining to confirm the osteogenic differentiation of BM-MSCs after 14 days of incubation in the presence of induction media. TCPS (control sample) and PLLA nanofibers and PLLA $+\mathrm{NI}$ Scaffold and NI (Nisin). (Scale bar is $100 \mu \mathrm{m}$ ) 

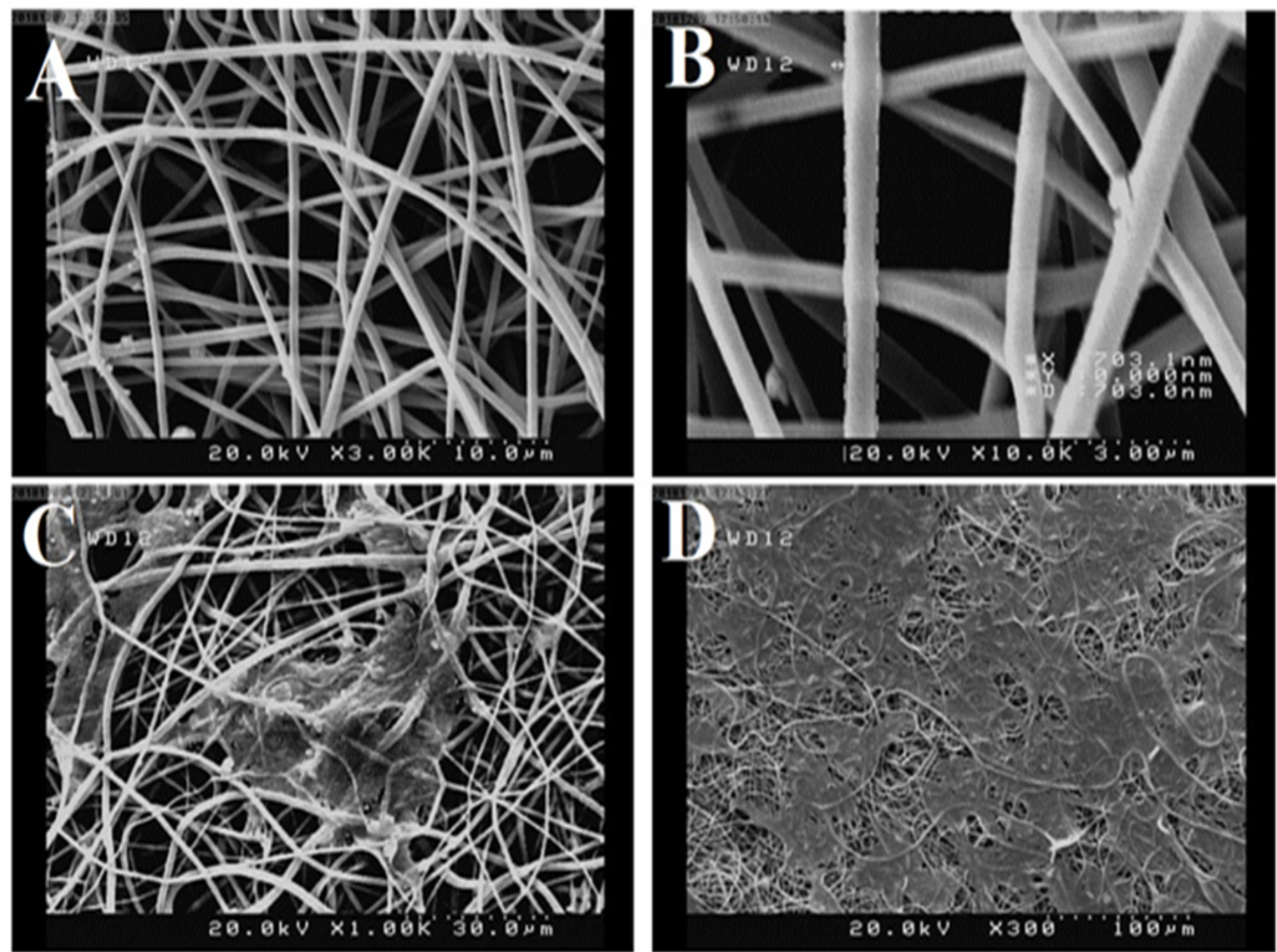

Fig. 6

Figure 6

PLLA Scafold images. A) Poly-L-lactic acid Scaffold without seeded cells, B) SEM microstructure of electrospun PLLA C) cell seeded on PLLA nanofibers scaffold D) cell seeded on PLLA nanofibers scaffold and Nisin 


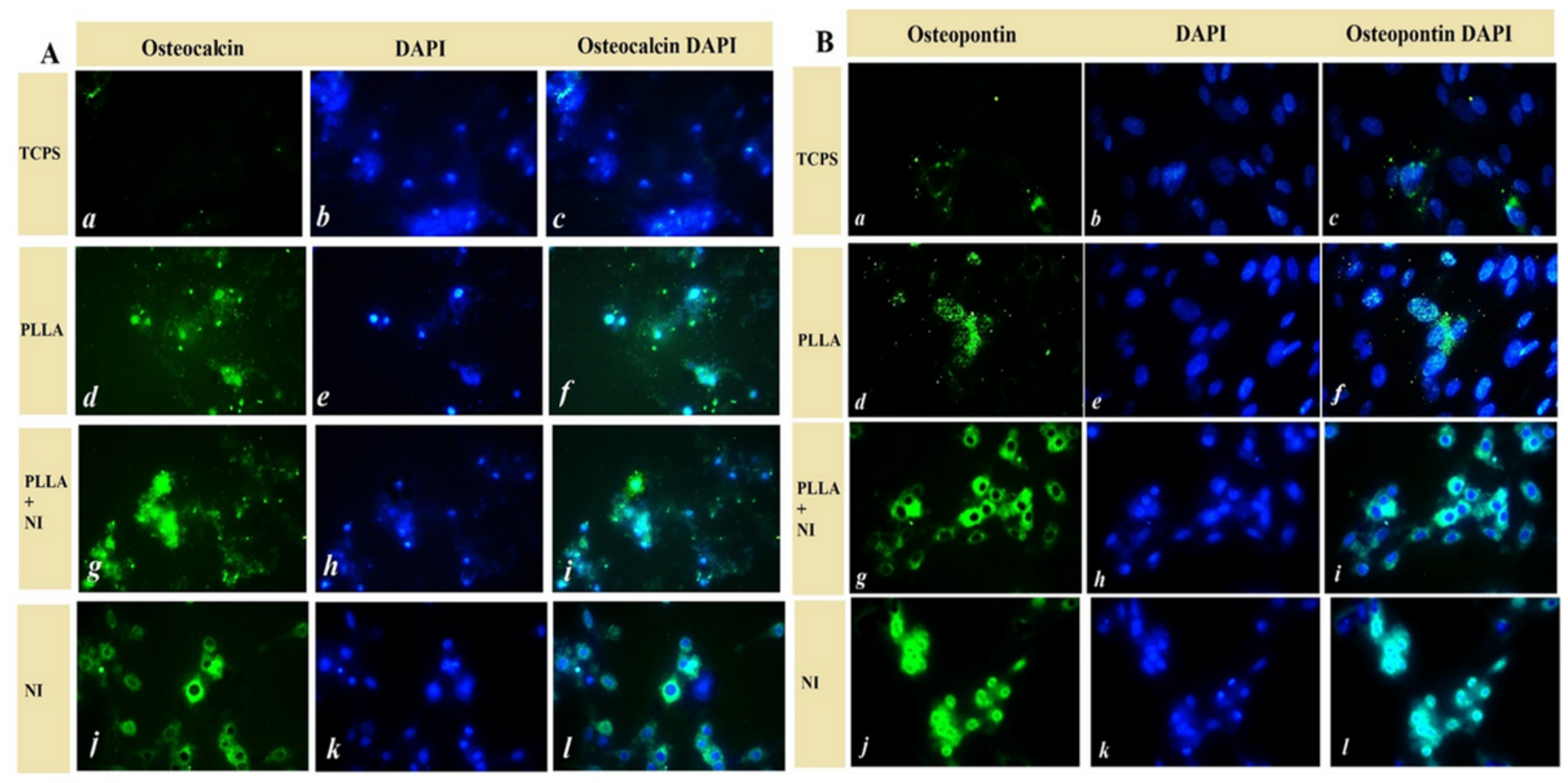

Fig. 7

Figure 7

Immunofluorescence staining of differentiated BM-MSCs on TCPS, A) Osteocalcin and B) Osteopontin, $(a, b, c), P L L A(d, e, f), P L L A+N I(g, h, i), N I(j, k, l)$ after 2 weeks. The cells were analyzed for the expression of osteogenic markers including Osteocalcin (a-l). DAPI staining (b,e,h,k) 


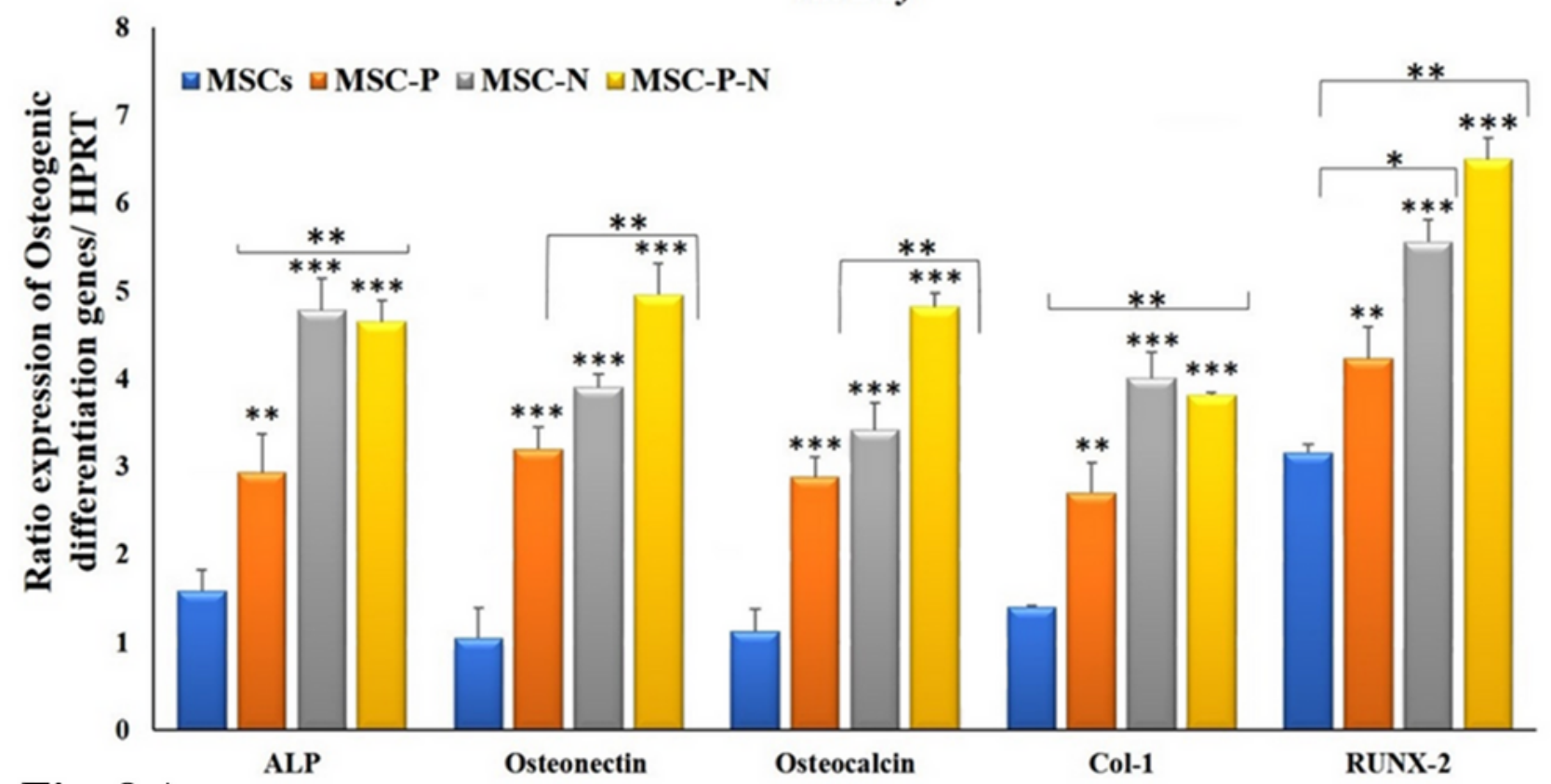

Fig. 8 A

\section{4th Day}

$\square$ MSCs $\square$ MSC-P $\square$ MSC-N $\square$ MSC-P-N

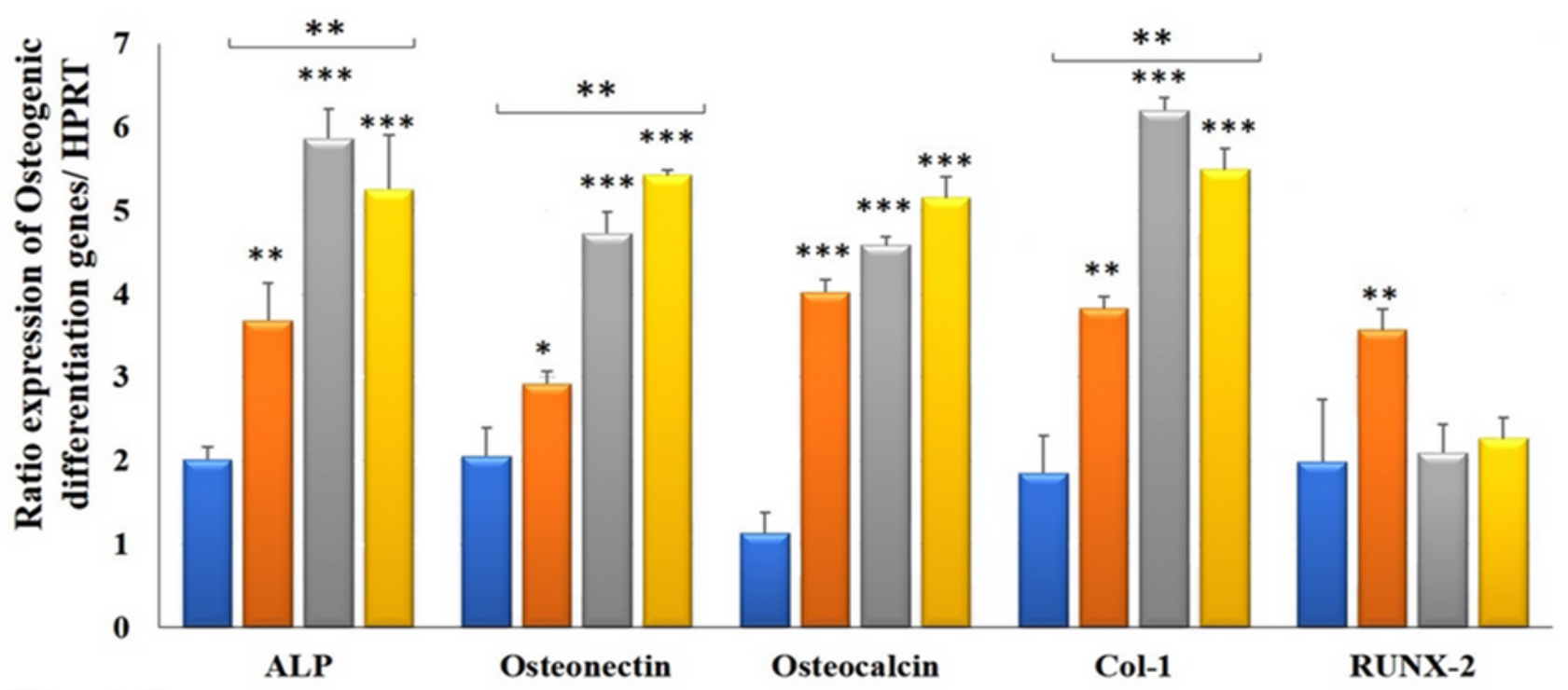

Fig. 8 B

\section{Figure 8}

Relative expression of ALP, Osteonectin, Osteocalcin, Col I, RUNX-2, A) on the day 7 and B) on the day 14, in BM-MSC on PLLA scaffold and Nisin during the osteogenic process. REST software was used for gene expression analysis using real-time PCR data from the rotor-gene Q. HPRT was used as a control for RNA sample quality. The results are presented as mean \pm SD. Significant levels are * $P \leq 0.05$ and $* \star P \leq 0.01$ and $* * * \mathrm{P} \leq 0.001$ 